Volume 1, Issue 2, Nov. - 2020: pp: 47-52 www.ejor.sohag-univ.edu.eg

Original article

\title{
CLINICAL, MRI, AND ARTHROSCOPIC CORRELATION INMENISCAL INJURIES OF THE KNEE
}

\author{
Khalid Abougabal $^{(*)}$, Moustafa Elsayed, Abdelrahman Khalifa, Ashraf Marzouk. \\ Orthopedic Surgery dept., Faculty of Medicine, Sohag Univ., Sohag, Egypt \\ *E-mail: Khalidgabal@icloud.com
}

Received 18/6/2020

Accepted 29/8/2020

\begin{abstract}
The aim of this study is to compare and correlate the clinical, magnetic resonance imaging (MRI), and arthroscopy findings in meniscal injuries of the knee. This was a prospective study of 30 cases of meniscal injuries of the knee admitted in Sohag University Hospital between January 2015 and June 2019, who underwent clinical examination, MRI, and arthroscopy of the knee. In our study of 30 cases, there were 24 male and six female patients with age ranging from 20 years to 39 years. Clinical examination had sensitivity of $86.9 \%$, specificity of $85.7 \%$, and accuracy of $86.6 \%$ for medial meniscus, and sensitivity of $57.2 \%$, specificity of $95.6 \%$, and accuracy of $86.6 \%$ for lateral meniscus. MRI had sensitivity of $95.6 \%$, specificity of $85.7 \%$, and accuracy of $93.33 \%$ for medial meniscus, and sensitivity of $85.7 \%$, specificity of $69.56 \%$, and accuracy of $73.33 \%$ for lateral meniscus. Clinical and MRI evaluations have no differences in the diagnosis of medial meniscus injuries. A trained radiologist obtained better sensitivity, specificity and accuracy in the diagnosis of lateral meniscus. Clinical diagnosis is of primary necessity. MRI is an additional diagnosing tool for meniscal injuries of the knee and can be used to exclude pathology, as the negative predictive value is high for all the lesions.
\end{abstract}

Keyword: Meniscal, Cruciate, Arthroscopy, Knee injuries, MRI

\section{Introduction}

The knee joint is a common site of injury due to trauma, repetitive activities, and sports activities. Clinical tests used in the diagnosis of meniscal injuries have limitations and it may be difficult to elicit objective signs repeatedly, mainly due to pain in an acute or sub acute presentation. History taking regarding the mechanism of knee injury gives a vital clue to the structures injured in the knee joint. Hyperextension with an audible pop would suggest an anterior cruciate ligament (ACL) tear. A direct blow to the knee from the side would point toward collateral ligament injury, and from the front, would indicate a cruciate ligament injury. Although clinical examination is most important for the diagnosis of a ligament injury, painful stress examinations are not always accurate in the acute phase of the injury. Clinical tests may be confusing and may cause a delay in diagnosis. Therefore, complementary diagnostic tools are often necessary, mainly when suspicion of multiple lesions exists. Magnetic resonance imaging (MRI) has a better soft tissue contrast and multiplanar slice capability which has revolutionized and has become the ideal modality for imaging the complex anatomy of the knee joint [1]. MRI is a completely noninvasive 
diagnostic modality and there is no ionizing radiation. Moreover, the ligaments of the knee are divided into intraarticular and extra articular. MRI plays a most important role in their evaluation. This division is important, as the extra articular ligaments are not visible on routine arthroscopic procedures. However, identification of meniscal tears can be difficult to interpret and can be observer dependent as well as dependent

\section{Materials and Methods}

This was a prospective study involving 30 patients with history of knee injuries who were admitted in the Department of Orthopaedics, Sohag University Hospital. Clinical examination and MRI of the knee joint was done for all these patients. The patients were then subjected to diagnostic and therapeutic arthroscopy by the arthroscopy team in the Department of Orthopaedics, Sohag University Hospital between January 2015 and June 2019. Patients included in this study were aged between 20 years and39 years, and had knee problems like pain, instability, and locking of the knee for 2-8weeks. Patients excluded from this study were those who had undergone previous meniscectomies, knee ligament repair or reconstructions and knee arthroscopies, anterior and posterior cruciate ligament injuries, knee joint neoplasm, infectious and inflammatory conditions of the knee joint, ferromagnetic implants, pacemakers, and aneurysm clips. Patients undergoing arthroscopy without MRI were also excluded from the study. All patients gave written consent for inclusion in the study. Complete examination of the knee was carried out, with particular emphasis on various tests. McMurray's test, joint line tenderness and Apley test were the diagnostic criteria considered for meniscal injuries. The tests for collateral ligament injuries were valgus/varus stress tests. The Lachman test, anterior drawer test, and posterior drawer test were used for identifying cruciate ligament tears X- upon the sensitivity of the scanner. Arthroscopy is considered as "the gold standard" for diagnosis of traumatic intraarticular knee lesions. Arthroscopy, being a highly sensitive and specific procedure, is both diagnostic and therapeutic, but is invasive and can cause complications like infection, haemarthrosis, adhesions, and reflex sympathetic dystrophy.

ray of the involved knee, anteroposterior (AP) and lateral views, was done to rule out any bony injury. MRI of the knee joint was done after 2 weeks post trauma and not immediately, in view of acute haemarthrosis or effusion of the knee, which would mask critical findings that would aid in diagnosis. Examination under anesthesia was done to confirm the signs of instability. Patients underwent arthroscopy. Clinical, MRI, and arthroscopy findings were recorded and compared. The composite data was tabulated and studied for correlation with clinical, MRI, and arthroscopic findings and grouped into four categories: (1) a result was considered to be true positive when the positive clinical or MRI diagnosis was confirmed by positive intra operative arthroscopic evaluation; (2) a result was considered to be true negative when the absence of pathological findings in clinical examination or MRI could be confirmed by arthroscopy; (3) a false positive result was defined as a positive clinical or MRI diagnosis with negative arthroscopy findings; and (4) a false negative result was defined as a positive intraoperative arthroscopy finding, but clinical or MRI diagnosis was found to be negative. Statistical analysis was used to calculate the sensitivity, specificity, positive predictive value (PPV), and the negative predictive value (NPV), in order to assess the reliability of clinical and MRI results. 


\section{Results}

In our study, 24 male patients and six female patients in the age group of 20-39 years were included. The right knee joint was found to be more commonly involved (20 patients) than the left knee joint (10 patients). Domestic fall was found to be the common mode of injury. Medial meniscus injury was more common than later a meniscus injury in our study. A total of 21 cases of medial meniscus injury were detected on clinical examination; arthroscopy confirmed only 20 cases. The sensitivity and specificity of clinical examination with respect to arthroscopy were $86.9 \%$ and $85.7 \%$, respectively, tab s. $_{\text {. }}(1 \& 2)$. MRI detected 22 cases of medial meniscus injury; arthroscopy confirmed only 21 cases. The sensitivity and specificity of MRI with respect to arthroscopy were
$95 \%$ and $85 \%$, respectively. 5 cases of lateral meniscus injury were detected on clinical examination; arthroscopy confirmed only 4 cases. The sensitivity and specificity of clinical examination with respect to arthroscopy were $57.2 \%$ and $95.6 \%$, respectively. In our study, MRI detected 13 cases of lateral meniscus injury; arthroscopy confirmed only 6 cases. The sensitivity and specificity of MRI with respect to arthroscopy were $85.7 \%$ and $69.56 \%$, respectively, tab. (3). Clinical examination and MRI had higher false positives in detecting meniscal tears. If MRI is used as the only form of preoperative screening for this condition, then there may well be unnecessary arthroscopies performed.

Table (1) True positive (TP), true negative (TN), false positive (FP) and false negative (FN) for each type of pathology

\begin{tabular}{|l|c|c|c|c|}
\hline & $\begin{array}{c}\text { Clinical exam } \\
\text { MM }\end{array}$ & $\begin{array}{c}\text { Clinical exam } \\
\text { LM }\end{array}$ & MRI of MM & MRI of LM \\
\hline True positive & 20 & 4 & 22 & 6 \\
\hline True negative & 6 & 22 & 6 & 16 \\
\hline False positive & 1 & 1 & 1 & 7 \\
\hline False negative & 3 & 3 & 1 & 1 \\
\hline
\end{tabular}

Table (2) Results for clinical examination in diagnosing meniscal tears

\begin{tabular}{|l|c|c|}
\hline & Medial meniscus & Lateral meniscus \\
\hline Sensitivity & 86.9 & 57.2 \\
\hline Specificity & 85.7 & 95.6 \\
\hline Positive predictive value & 95.2 & 80 \\
\hline Negative predictive value & 66.66 & 88 \\
\hline Accuracy & 86.6 & 86.6 \\
\hline
\end{tabular}

Table (3) Results for magnetic resonance imaging (MRI) in diagnosing meniscal tears

\begin{tabular}{|l|c|c|}
\hline & Medial meniscus & Lateral meniscus \\
\hline Sensitivity & 95.6 & 87.7 \\
\hline Specificity & 85.7 & 69.56 \\
\hline Positive predictive value & 95.6 & 46.15 \\
\hline Negative predictive value & 85.7 & 94.11 \\
\hline Accuracy & 93.33 & 73.33 \\
\hline
\end{tabular}

\section{Discussion}

The purpose of this study was to compare the accuracy of clinical and MRI findings in diagnosing the meniscal and ligamentous injuries of chronic painful knees. In the present study of 30 patients,
24 were males and six were females. The age ranged from 20 years to 39 years. In the present study, males comprised the predominant number of patients who suffered knee injuries, which were mainly 
due to domestic falls. The right knee was involved in 20 cases $(66.6 \%)$ and the left knee was involved in 10 cases $(33.3 \%)$; there was no bilateral involvement. Mackenzie et al [2] studied 332 patients' diagnosis before and after MRI. The diagnosis was initially based on the clinical examination and the therapeutic procedure was decided before MRI. A total of 57 from 113 clinically positive before MRI meniscal tears were not confirmed with MRI. This result led to revaluation and differentiation of treatment in $62 \%$ of the patients. From those patients programmed for surgery, only $38 \%$ finally underwent arthroscopy. Weinstabl et al [3] randomly distributed patients with positive meniscus rupture tests into two groups. All of the patients in the first group had MRI examination before arthroscopy. In this group, only $2 \%$ of patients did not have positive findings during arthroscopy. The second group of patients underwent arthroscopy, based only on the findings of clinical examination. In this group, arthroscopy confirmed the findings of clinical examination in only $30 \%$ of patients. The sensitivity for diagnosing isolated medial meniscal tears in a series by Rubin et al [4] was $98 \%$ and it decreased when other structures were also injured. The specificity in isolated lesions was $90 \%$. In a multi centric analysis, Fisher et al [5] reported an accuracy of $78-97 \%$ for the ACL and $64-95 \%$ for medial meniscus tears. The meniscus is composed of fibro cartilage and appear as low signal structures on all pulse sequences. The sensitivity and specificity of MRI in detecting meniscal tears exceeds $90 \%$ [6]. Simultaneous injury to several supporting structures is relatively common in the knee. When more than one lesion was present, completely correct diagnosis was rendered only $30 \%$ of the time. This phenomenon was reported by Rubin et al. [4]. In a prospective study reported by Imhoff et al. [7], the NPV was $94 \%$ but the PPV was only 54\%. They concluded that due to a high NPV, a normal MRI scan allows eliminating a meniscal lesion and so there is no need for a diagnostic arthroscopy. They suggested that due to the low PPV of MRI, it should not be routinely used to confirm clinical diagnosis and its use should be limited to those cases where clinical examination is inconclusive. A diagnostic arthroscopy would be a better choice in those cases. However, in our study, MRI showed false results in a significant proportion. For example, as far as medial meniscus was concerned, there were one false positive diagnoses and one false negative diagnosis, whereas for lateral meniscus, there were seven false positive diagnoses and one false negative diagnoses (PPV 95\% and46\%; NPV $85.7 \%$ and $94.11 \%$, for medial meniscus and lateral meniscus tears, respectively). Disruption of the ACL, a major stabilizer of the knee, leads to loss of stability of the knee and potentially significant dysfunction. Although the ACL is the most frequently torn ligament of the knee, the ACL tear has remained clinically elusive. These injuries account for a large number of referrals to hospitals. The evaluation of these lesions remains a difficult clinical problem. MRI is a frequently used diagnostic modality for these internal derangements because of being noninvasive, painless, and not associated with the risk of radiation. Arthroscopy is a technically demanding procedure and the results vary according to the surgeon's experience, especially in difficult cases. Majority of the false positive results refer to a posterior meniscus tear. Nevertheless, the belief is that, even in these cases, the meniscal pathology existed but failed to be discovered during arthroscopy $[12,13]$. In particular, the inferior surface of the posterior aspect of the medial meniscus is difficult to reach with a probe and often ruptures at that point can be missed. Nowadays, the overall accuracy of arthroscopy varies between $70 \%$ and $100 \%$, depending on the surgeon's experience [14-17]. This fluctuation inevitably raises questions regarding the reliability of the MRI results classification on true 
or false [18]. In everyday practice, based on clinical examination coming first, surgeons decide whether to proceed to further laboratory tests, MRI, or conservative or surgical treatment. However, how precise can clinical examination be? There seems to be disagree men regarding the answer to this question. Investigations support the fact that the accuracy of clinical examination compared with arthroscopic findings ranges between $64 \%$ and $85 \%[19,20]$. Rose and Gold [9] found that clinical examination is as accurate as MRI in diagnosing meniscal tears and ACL ruptures, so they concluded that MRI, because of its high cost, is not necessary in patients with clinical suspicion of meniscus and cruciate ligament tears. A similar conclusion was reported by Boden et al [21] who supported that when clinical examination sets the diagnosis of meniscus damage, MRI will not change treatment decisions. Jackson et al [22] concluded that negative MRI for meniscus and cruciate ligament tears can discourage diagnostic arthroscopy, even if clinical examination is positive for injury.

\section{Conclusion}

By obtaining correlation between clinical examination, MRI scan, and arthroscopy for meniscal injuries, we conclude that carefully performed clinical examination can give equal or better diagnosis of meniscal injuries in comparison to MRI scan. MRI scan may be used to rule out such injuries rather than to diagnose them. MRI scan has a much better negative predictive value than positive predictive value in meniscal injury diagnosis. When clinical signs and symptoms are inconclusive, performing an MRI scan is likely to be more beneficial in avoiding unnecessary arthroscopic surgery. When clinical diagnosis is in favor of meniscal injuries, performing an MRI scan prior to arthroscopic examination is unlikely to be of significance. MRI scanning should not be used as a primary diagnostic tool in meniscal injuries. By passing MRI scans and performing arthroscopic examination in suspected cases will be helpful providing earlier treatment of the condition.

\section{References}

[1] Kaplan, P., Walker, C, Kilcoyne, R., et al. (1992). Occult fracture patterns of the knee associated with ACL tears: Assessment with MR imaging. Radiology. 183: 835-838.

[2] Mackenzie, R., Dixon, A., Keene, G., et al. (1996). Magnetic resonance imaging of the knee; assessment of the effectiveness. ClinRadiol. 51: 245-250.

[3] Weinstabl, R., Muellner, T., Vecsei, V, et al. (1997). Economic considerations for the diagnosis and therapy of meniscal lesions: Can MR imaging help reduce the expense?. World J Surg. 21: 363-368.

[4] Rubin, D., Kettering, J., Towers, J., et al. (1998). MR imaging of knee having isolated and combined ligament injuries. Am J Roentgenol. 170: 1207-1213.

[5] Fisher, S., Fox J., Del Pizzo, W., et al. (1991). Accuracy of diagnosis from magnetic resonance imaging of the knee; a multicentric analysis of one thousand and fourteen patients. J Bone Joint Surg Am. 73: 2-10.

[6] Miller, R. Knee Injuries. (1998). In: Terry, C., (ed.) Campbell's operative orthopaedics, St Louis, Mosby, pp. 1113-1299.

[7] Imhoff, A., Buess, E., Holder, J., et al. (1997). Comparison between magnetic resonance imaging and arthroscopy for the diagnosis of knee meniscal lesion.

Rev Chir Orthop. 83: 229-236

[8] Hodler, J., Haghighi, P., Trudell, D., et al. (1992). The cruciate ligaments of the knee: Correlation between MR appearance and gross and histologic findings in cadaveric specimens. $\mathbf{A m}$ J Roentgenol. 159: 357-360.

[9] Rose, N., Gold, S. (1996). A comparison of accuracy between clinical examin- 
ation and magnetic resonance imaging in the diagnosis of meniscal and anterior cruciate ligament tears. Arthroscopy. 12: 398-405.

[10]Lee, J., Yao, L., Phelps, C., et al. (1998). Anterior cruciate ligament tears: MR imaging compared with arthroscopy and clinical tests. Radiology. 166: 861-864.

[11]Mink, J., Levy, T., (1988). Crues $3^{\text {rd }}$ JV. Tears of the anterior cruciate ligament and menisci of the knee: MR imaging evaluation. Radiology. 167: 769-774.

[12]Spiers, A., Meagher, T., Ostlere, S., et al. (1993). Can MRI of the knee affect arthroscopic practice? A prospective study of 58 patients. J Bone Joint Surg Br. 75: 49-52.

[13]Crues III, J., Mink, J., Levy, T., et al. (1987). Meniscal tears of the knee: Accuracy of MR imaging. Radiology. 164: 445-448.

[14]Ireland, J., Trickey, E., Stoker, D. (1980). Arthroscopy and arthrography of the knee: A critical review. J Bone Joint Surg Br. 62: 3-6.

[15]Dandy, D., Jackson, R. (1975). The diagnosis of problems after menisciectomy. J Bone Joint Surg Br. 57: 349-352.
[16]Jackson, R., Abe, I. (1972). The role of arthroscopy in the management of disorders of the knee: An analysis of 200 consecutive examinations. J Bone Joint Surg Br. 54: 310-322.

[17]Boeve, B., Davidson, R., Staab Jr. (1991). Magnetic resonance imaging in the evaluation of knee injuries. South Med J. 84: 1123-1127.

[18]Mackenzie, R., Keene, G., Lomas, D., et al. (1995). Errors at knee magnetic resonance imaging: true or false? $\mathbf{B r}$ J Radiol. 68: 1045-1051.

[19]Gillies, H., Seligson, D. (1979). Precision in the diagnosis of meniscal lesions: A comparison of clinical evaluation, arthrography, and arthroscopy. J Bone Joint Surg Am. 61: 343-346.

[20]Simonsen, O., Jensen, J., Mouritsen, P., et al. (1984). The accuracy of clinical examination of injury of the knee joint. Injury. 16: 96-101.

[21]Boden, S., Labropoulos, P., Vailas, J. (1990). MR scanning of the acutely injured knee: Sensitive, but is it cost effective? Arthroscopy. 6: 3063-10.

[22]Jackson, D., Jennings, L., Maywood, R., et al. (1988). Magnetic resonance imaging of the knee. Am J Sports Med. 16: 29-38. 